\title{
Development of a CT scanner based on the Medipix family of detectors
}

\author{
Philip J. Bones ${ }^{a}$, Anthony P.H. Butler ${ }^{b}$, J. Paul Ronaldson ${ }^{b}$ and Alex M.T. Opie ${ }^{a}$ \\ ${ }^{a}$ Computational Imaging Group, Department of Electrical \& Computer Engineering, \\ University of Canterbury, Christchurch, New Zealand. \\ ${ }^{b}$ Department of Radiology, University of Otago, Christchurch, New Zealand.
}

\begin{abstract}
Photon counting detectors are of growing importance in medical imaging because they enable routine measurement of photon energy. Detectors such as Medipix2 and Medipix3 record the energy of incident photons with minimal loss of spatial resolution. Their use is being investigated for both pre-clinical and clinical applications of X-ray CT. The Medipix3 detector has 256 x $25655 \mu \mathrm{m}$ pixels and a silicon or cadmium telluride detector layer, giving a spatial resolution comparable to mammographic film. Each Medipix pixel can be seen as an individual spectral detector. The logic circuits for each pixel (some 1300 transistors) can analyze incoming events at megahertz rates, comparing the charge of the electron-hole cloud with preset levels, giving a resolution of about $2 \mathrm{keV}$ across the range of $8-140 \mathrm{keV}$.

A prototype CT scanner has been developed for laboratory animals and excised specimens. Applications under investigation include: K-edge imaging: Using spectral information to measure heavy elements (e.g., preparations of iodine, barium, and gadolinium) and Soft tissue contrast: Dual energy systems have shown that image contrast for soft tissue can be improved, e.g., distinguishing between iron and calcium within vascular plaques.
\end{abstract}

\section{INTRODUCTION}

Spectroscopic x-ray detectors, such as Medipix, ${ }^{1,2}$ are opening the door to the widespread use of energy selective biomedical x-ray imaging. With dual energy computed tomography rapidly establishing itself as the clinical standard for many radiological investigations, spectroscopic imaging is a likely next step. However to confirm the utility of spectroscopic x-ray detectors there needs to be a clearer indication of the clinical benefits of the technology.

Some 15 years ago the first Medipix Collaboration was formed to transfer the ideas generated in designing detectors to characterize the jets of particles created in $14 \mathrm{GeV}$ proton-on-proton collisions (for the CERN Large Hadron Collider), to medical and industrial applications. The University of Canterbury is a foundation member of the Medipix3 collaboration. The Medipix3 2D imaging detector ${ }^{2}$ has the ability to count photon events within up to 8 separate energy bands and the ability to arbitrate between neighboring pixels to decide to which a particular photon event belongs (charge summing mode). In single pixel mode, the detector resolution is $55 \mu \mathrm{m}$.

In order to identify possible applications of spectroscopic imaging we conducted a literature review of clinical applications of dual energy systems. ${ }^{3-7}$ In addition we analyzed simulation results from our own group, our collaborating partners, and industry. ${ }^{8-13}$

We expect that the benefits of spectroscopic x-ray imaging lie in three areas:

1. Improved image quality, in particular a reduction in beam hardening artefact and scatter reduction. ${ }^{14,15}$

2. K-edge imaging, i.e., the identification of high Z-number contrast agents based on their k-edge. Potential advantages include a) less contrast agent required in high risk patients such as those with renal impairment, diabetes, or the elderly, and b) separation of several contrast agents allowing for multi-phase studies to be performed with a lower x-ray dose and less time on the scanner. ${ }^{13,16,17}$

Further author information:

Philip J. Bones: E-mail: phil.bones@canterbury.ac.nz, Telephone: +64 33642987 ext. 7275 
3. Improved soft tissue contrast from differences in mass attenuation coefficients of different tissues. The diagnoses of several diseases, such as breast cancer, are known to be improved by dual energy systems. In addition, there is improved soft tissue contrast of normal structures. ${ }^{12,18,19}$

These potential benefits need to be weighed up with the clinical significance of diseases and current radiological practice. Our own efforts to evaluate the potential for spectral CT have included using principal components analysis to separate regions of different attenuation characteristics, ${ }^{10}$ distinguishing different contrast agents in an animal preparation, ${ }^{11,17}$ and distinguishing soft tissue types. ${ }^{12}$ In this paper we review the underlying technology, the efforts taken to date at analyzing spectral CT outputs, and summarize two specific applications. Two results are presented in support of our conclusions that spectral CT has a promising future.

\section{MEDIPIX DETECTOR}

The Medipix detector is a by-product of the Large Hadron Collider project at CERN. A small company was established to design and build the detector and a large collaboration of universities and research institutes has been formed to develop applications and accessories for the detector family. A number of studies have employed the Medipix2. ${ }^{1}$ The current state of development is the Medipix3.

Both silicon ( $\mathrm{Si}$ ) and cadmium telluride (CdTe) detector layers have been tested on prototype Medipix sensors. Substantially higher energy photons can be imaged with the CdTe detector, so it seems that the broader spectrum that can thereby be exploited will make it the material of choice for the imaging of biological specimens.

\subsection{Medipix3}

The Medipix chip is $14 \mathrm{~mm} \times 14 \mathrm{~mm}$ and comprises $256 \times 256$ square pixels, each of edge length $55 \mu \mathrm{m}^{2}{ }^{2}$ The Medipix 3 prototype chip has been designed and manufactured in $0.13 \mu \mathrm{m}$ CMOS technology with eight metal layers. This allows a high degree of interconnectivity between pixels and the implementation of more functionality while maintaining a compact pixel area. New features on the Medipix3 compared to its predecessors include the ability to arbitrate which pixel is assigned an x-ray photon which strikes the detector in between pixel centers and the ability to distinguish 8 energies simultaneously with $110 \mu \mathrm{m}$ resolution.

\section{MULTIPLE ENERGIES IN CT}

We see the work of Alvarez \& Macovski (1976) ${ }^{14}$ who used two energies to find coefficients for two basis functions, as seminal in the field of exploiting energy sensitive detectors. The basis functions proposed by Alvarez \& Macovski were each modeling one of the primary attenuation processes: photoelectric interactions and Compton scattering. As well as establishing an elegant framework for analysing multi-energy systems, they showed a simulated example that resolved fatty tissue in the brain, comparing a standard reconstruction and the reconstructions of each of their two coefficients. A number of other researchers have used Alvarez and Macovski's basis functions in their work and in some cases extended the fundamental idea with additional functions. ${ }^{4,16,20}$

Roessl \& Proksa $(2007)^{16}$ were primarily interested in imaging of k-edge materials. This paper contains a simulated scan where they need to distinguish between a gadolinium-based contrast agent and an atherosclerotic plaque, where they claim that conventional integrating systems struggle or fail to differentiate between the two materials while their energy-selective system shows a strong contrast between them.

Schlomka et $a l^{21}$ concentrate on k-edge imaging. This paper is the experimental follow-up to Roessl \& Proksa. ${ }^{16}$ They have successfully managed to extend Alvarez and Macovski's ${ }^{14}$ two basis functions to four, and identify iodine and gadolinium within a phantom containing both. Interestingly, their choice of four basis functions were: photoelectric absorption, Compton scattering, attenuation by iodine, and attenuation by gadolinium. The functions describing the latter two were designed to model the total attenuation caused by these elements.

The energy weighting technique for using spectral information has received a lot of attention. The original work seems to have been that of Tapiovaara \& Wagner (1985). ${ }^{22}$ A simple two-material model is adopted to derive optimal weightings for each energy bin based on maximizing the SNR. Niederlöhner et $a l^{8,23}$ report that their simulations predict an SNR improvement of a factor up to 1.5 compared to a conventional integrating detector.

Taguchi et al have recently studied the effects of pulse pileup, which is likely to occur if flux rates are high. ${ }^{24}$ 


\subsection{Principal components analysis}

Our own efforts at reconstructing CT images from multiple energy recordings have centered on the use of principal components analysis (PCA). ${ }^{10}$ The goal of PCA is to identify linearly independent patterns of variance within a data set. PCA can be applied to object projections, as it was in Butzer et al, ${ }^{10}$ or to reconstructed $3 \mathrm{D}$ data. ${ }^{17}$ With PCA, eigenimages can be formed for each of the largest eigenvalues and these correspond to strong substance types in the data. Thus an implicit model for the energy dependence is built, based on the data, rather than one based on a model.

\section{APPLICATIONS}

Biomedical applications for the Medipix-based CT scanner under investigation can broadly be grouped into those that rely on radiographic pharmaceuticals with suitable k-edges and those based on the intrinsic contrast of tissues.

\subsection{K-edge imaging}

Extrinsic radiological pharmaceuticals used for human, small animal, or pathology specimens commonly have heavy elements. These elements have an photoelectric absorption edge that can be measured by looking at the energy specific attenuation above and below the k-edge or l-edge.

For clinical imaging the choice of atom depends on the absorption edge being within a suitable x-ray energy range, typically 30-120 keV, and the possibility of developing a tolerated pharmaceutical. For pre-clinical experiments where the specimen or animal tends to be smaller and/or non-living these requirements can be relaxed, allowing the use of a greater range of pharmaceutical products. In clinical practice the heavy atoms used include iodine, gadolinium, and barium. In small animal imaging and specimen imaging the list is extended to include gold, silver, lead, and many others.

Being able to identify and quantify pharmaceutical agents allows several contrast agents to be used in conjunction. ${ }^{11,13,17}$ Combinations in different body spaces or different timing of infusions can then yield information on complex anatomy or physiology. For example, one pharmaceutical could be infused into a vessel's lumen and a different agent introduced into the vaso vasorum of the vessel wall.

Another application of k-edge imaging in pre-clinical investigations is atomic substitution. It has been known for a long time that many light elements found in normal physiology can be substituted with heavier elements. ${ }^{25,26}$ Typically, substitution involves replacing a light element with a heavier element with a similar outer electron configuration. Thus the heavier element has similar chemical properties but better x-ray attenuation properties. Published examples include substituting strontium (k-edge $16 \mathrm{keV}$ ) for physiological calcium and rubidium (kedge $15 \mathrm{keV}$ ) for physiological potassium. In these substitutions the lighter elements have very low k-edges that are not suitable for imaging while the heavier elements have k-edges in the range used for imaging small objects.

There are many groups in the world developing nano-particles and other functional pharmaceuticals. These agents often contain gold or other heavy elements. ${ }^{27,28}$ Currently, our institute is developing simple gold containing lipid spheres of consistent size to measure permeability of sinusoids within the liver.

\subsection{Intrinsic contrast}

Different tissues of the body have different energy-specific attenuation profiles (linear attenuation coefficients). This has been exploited for dual energy CT and applications under investigation or of proven clinical utility include renal stone characterization and bone removal. ${ }^{18-20}$ While improved intrinsic tissue contrast is very promising, with the use of dual energy CT technology applications have been limited by noise and/or x-ray dose. This is because the dual energy CT usually involves dual exposures with two overlapping source x-ray spectra.

A clinically very important example of the need for intrinsic tissue contrast is in differentiating iron and calcium within vascular atheroma plaques. ${ }^{29}$ The strong clinical need is to identify plaques which are likely to rupture, causing stroke or heart attack. While this is a challenging application for energy resolved imaging, it is tantalisingly close with dual energy CT. It is hoped that the improved energy information from spectral photon counting CT systems may address this clinical need. 


\section{RESULTS}

The results presented here are indicative only of the work performed to date. Please see Butzer et al (2008), ${ }^{10}$ Berg et al (2009), ${ }^{12}$ and Anderson et al $(2010)^{17}$ for other examples from the group.

In Fig. 1 the measured response of a prototype Medipix3 sensor with a Si detector layer has been characterized with respect to direct ("open" beam) exposure to a small x-ray source at $50 \mathrm{keV}$. A complete spectrum has been obtained by making repeated measurements with different energy threshold settings for the Medipix3. The dominant effect of charge sharing at low energies which is evident in Fig. 1(a) is virtually removed when the charge summing mode (CSM) is invoked (Fig. 1(b)). The residual peak at low energies in Fig. 1(b) is largely contributed by electronic noise.

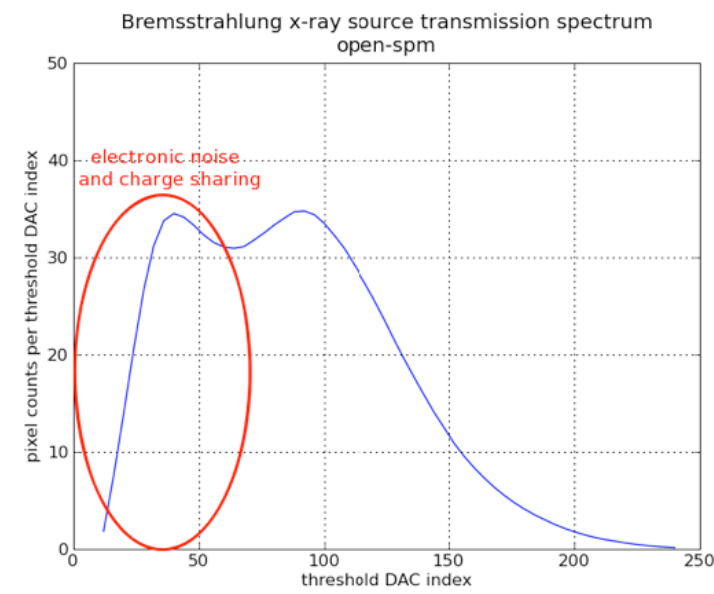

(a)

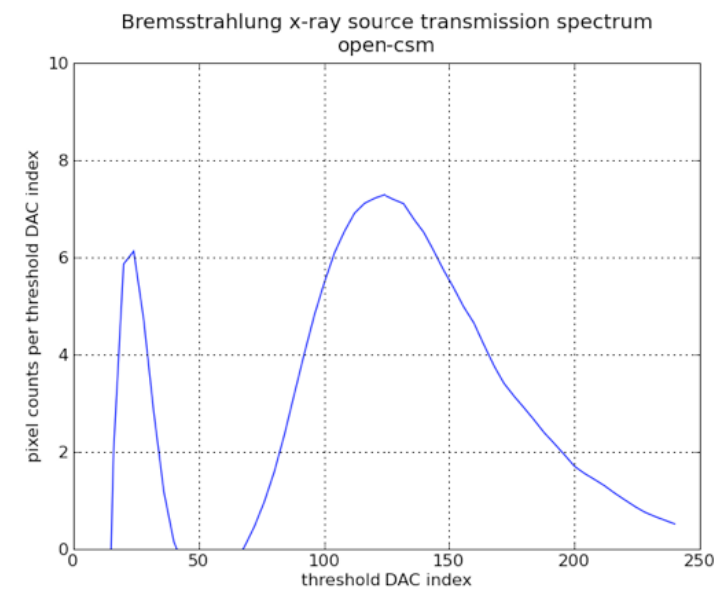

(b)

Figure 1. Response of a Medipix3 with Si detection layer. The Source Ray Inc. x-ray tube has intrinsic filtration of 1.8 $\mathrm{mm} \mathrm{Al}$ (equivalent). (a) In the single pixel mode (SPM), charge sharing effects dominate at lower energies. (b) This effect is remedied by the application of charge summing mode (CSM). The working range of this configuration of Medipix3 is for $E \sim 15-50 \mathrm{keV}$ (corresponding to DAC values $\sim 70-200$ ).

In Fig. 2, the results of imaging with a Medipix2 sensor with a CdTe detector layer are shown. The object imaged was a phantom comprising cylindrical holes in a perspex block, each filled will a different contrast substance. The relative location of the cylinders is shown in Fig. 2(a). A set of repeated measurements with different energy threshold settings for the Medipix2 were made. Then planar tomographic reconstructions were made by filtered back projection and the images combined by PCA to identify regions with attenuation of differing energy dependence. The resulting coloured image is shown in Fig. 2(b). There is a clear difference shown between the iodine, barium and gadolinium regions and each of these is different to the reference water-filled cylinder.

\section{CONCLUSIONS}

Spectral CT, using energy discriminating detectors like Medipix, seems a natural successor to dual-energy CT in medical imaging. However, the increase in complexity involved must be motivated by proven clinical utility. Evidence is accumulating that intrinsic tissue contrast may be improved with spectral CT. The high efficiency afforded by the charge summing feature of Medipix3 promises to allow dose reduction, while early results support the conjecture that spectral CT will allow new diagnostic methods employing multiple contrast agents.

\section{ACKNOWLEDGEMENTS}

The authors wish to acknowledge the contributions and encouragement of the Medipix2 and Medipix3 Collaborations (http://medipix.web.cern.ch/MEDIPIX/). We thank in particular Dr Michael Fiederle (Albert-LudwigsUniversität Freiburg) for supplying the CdTe used in forming Fig. 2(b). 


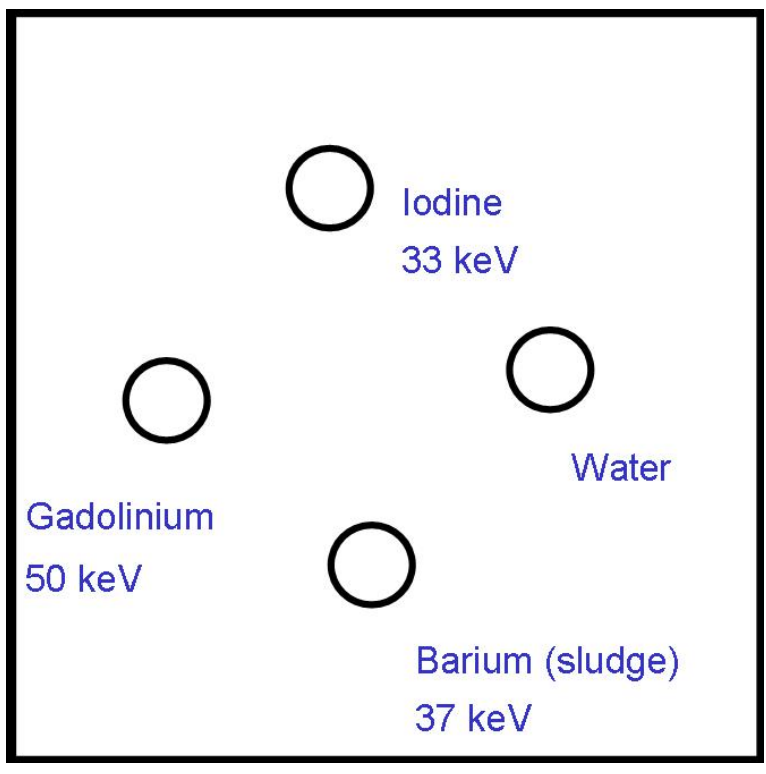

(a)

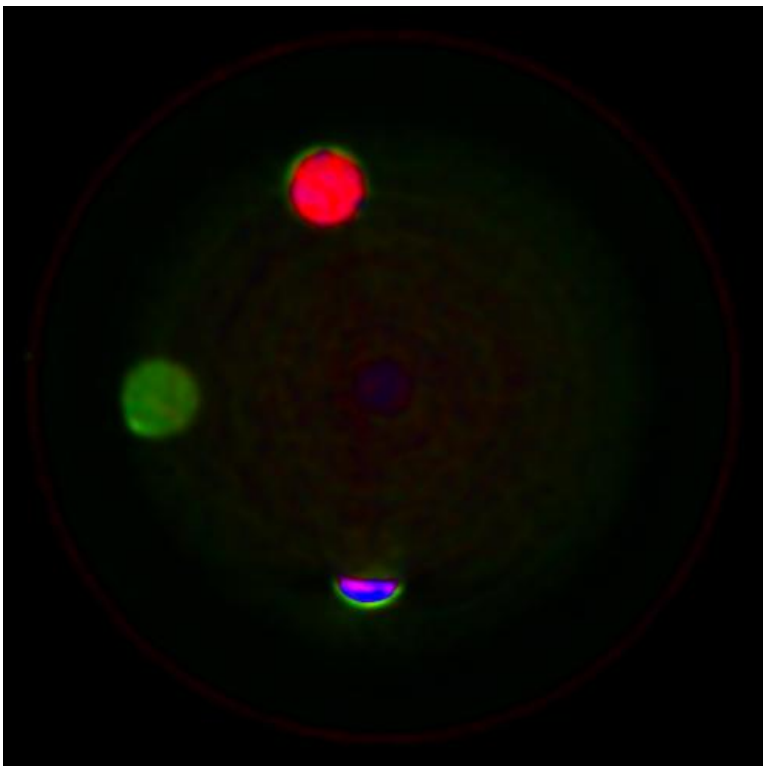

(b)

Figure 2. An image formed by imaging with a CdTe detector layer. (a) The key to the cross-section of the phantom which comprised a perspex block with 4 cylindrical holes, each filled with a different contrast medium. (b) A tomogram formed by reconstructing from projections and using PCA to separate different absorption versus energy characteristics of the contrast media. When the image is viewed in colour, the iodine appears red, the gadolinium green, the barium blue/pink, and the water dark blue.

\section{REFERENCES}

1. Llopart, X., Campbell, M., Dinapoli, R., Segundo, D. S., and Pernigotti, E., "Medipix2: A 64-k pixel readout chip with $55-\mu \mathrm{m}$ square elements working in single photon counting mode," IEEE Trans. Nucl. Sci. 49(5), 2279-2283 (2002).

2. Ballabriga, R., Campbell, M., Heijne, E. H. M., Llopart, X., and Tlustos, L., "The Medipix3 prototype, a pixel readout chip working in single photon counting mode with improved spectrometric performance," IEEE Trans. Nucl. Sci. 54(5), 1824-1829 (2007).

3. Johns, P., Drost, D., Yaffe, M., and Fenster, A., "Dual-energy mammography: Initial experimental results," Med. Phys. 12(3), 297-304 (1985).

4. Harding, G. and Tischler, R., "Dual-energy compton scatter tomography," Phys. Med. Biol. 31(5), 477-489 (1986).

5. Fessler, J. A., Elbakri, I., Sukovic, P., and Clinthorne, N., "Maximium-likelihood dual-energy tomographic image reconstruction," in [Proc. SPIE, Medical Imaging], 4684, 38-49, SPIE (2002).

6. Maaß, C., Baer, M., and Kachelrieß, M., "Image-based dual energy ct using optimized precorrection functions: a practical new approach of material decomposition in image domain," Med. Phys. 36(8), 3818-3829 (2009).

7. Szczykutowicz, T. P., Hsieh, J., and Chen, G., "The dependence of image quality on the number of high and low $\mathrm{kVp}$ projections in dual energy $\mathrm{CT}$ using the prior image constrained compressed sensing (PICCS) algorithm," in [Proc. SPIE, Medical Imaging], 7622, 762221, SPIE (2010).

8. Niederlöhner, D., Karg, J., Giersch, J., and Anton, G., "The energy weighting technique: measurements and simulations," Nucl. Instrum. Meth. Phys. Res. A 546, 37-41 (2005).

9. Butler, A., Anderson, N., Tipples, R., Cook, N., Watts, R., Meyer, J., Bell, A., Melzer, T., and Butler, P., "Bio-medical X-ray imaging with spectroscopic pixel detectors," Nucl. Instrum. Meth. Phys. Res. A 591, 141-146 (2008). 
10. Butzer, J. S., Butler, A., Butler, P., Bones, P., Cook, N., and Tlustos, L., "Medipix imaging - evaluation of datasets with PCA," in [Proc. Im. Vis. Comp. NZ], 1-6 (2008).

11. Firsching, M., Butler, A., Scott, N., Anderson, N., Michel, T., and Anton, G., "Contrast agent recognition in small animal CT using the medipix2 detector," Nucl. Instrum. Meth. Phys. Res. A 607, 179-182 (2009).

12. Berg, K., Carr, J., Clark, M., Cook, N., Anderson, N., Scott, N., Butler, A., and Butler, P., "Pilot study to confirm that fat and liver can be distinguished by spectroscopic tissue response on a medipix-all-resolutionsystem-CT (MARS-CT)," in [Proc. Adv. Mater. Nano.], 1151, 106-110, Amer. Inst. Phys. (2009).

13. Firsching, M., Giersch, J., Niederlöhner, D., and Anton, G., "A method for stoichiometric material reconstruction with spectroscopic x-ray pixel detectors," in [Proc. IEEE Nucl. Sci. Symp.], 4116-4119, IEEE (2004).

14. Alvarez, R. E. and Macovski, A., "Energy-selective reconstructions in x-ray computerized tomography," Phys. Med. Biol. 21(5), 733-744 (1976).

15. Shikhaliev, P., "Beam hardening artefacts in computed tomography with photon counting, charge integrating and energy weighting detectors: a simulation study," Phys. Med. Biol. 50, 5813-5827 (2005).

16. Roessl, E. and Proksa, R., "K-edge imaging in x-ray computed tomography using multi-bin photon counting detectors," Phys. Med. Biol. 52, 4679-4696 (2007).

17. Anderson, N., Butler, A., Scott, N., Cook, N., Butzer, J., Schleich, N., Firsching, M., Grasset, R., de Ruiter, N., Campbell, M., and Butler, P., "Spectroscopic (multi-energy) CT distinguishes iodine and barium contrast material in mice," Eur. Radiol. online, 1-9 (2010).

18. Johns, P. and Yaffe, M., "X-ray characterisation of normal and neoplastic breast tissues," Phys. Med. Biol. 32(6), 675-695 (1987).

19. Lemacks, M., Kappadath, S., Shaw, C., Liu, X., and Whitman, G., "A dual-energy subtraction technique for microcalcification imaging in digital mammography: A signal-to-noise analysis," Med. Phys. 29(8), 1739-1751 (2002).

20. Alvarez, R. E., "Near optimal energy selective x-ray imaging system performance with simple detectors," Medical physics 37(2), 822-841 (2010).

21. Schlomka, J. P., Roessl, E., Dorscheid, R., Dill, S., Martens, G., Istel, T., Bäumer, C., Herrmann, C., Steadman, R., Zeitler, G., Livne, A., and Proska, R., "Experimental feasibility of multi-energy photoncounting K-edge imaging in pre-clinical computed tomography," Phys. Med. Biol. 53, 4031-4047 (2008).

22. Tapiovaara, M. J. and Wagner, R., "SNR and DQE analysis of broad spectrum x-ray imaging," Phys. Med. Biol. 30(6), 519-529 (1985).

23. Niederlöhner, D., Giersch, J., Firsching, M., and Anton, G., "Practical aspects of energy weighting in x-ray imaging," in [Nuclear Science Symposium Conference Record], 5, 3191-3194 (2004).

24. Taguchi, K., Frey, E., and Wang, X., "An analytical model of the effects of pulse pileup on the energy spectrum recorded by energy resolved photon counting x-ray detectors," Med. Phys. 37(8), 3957-3969 (2010).

25. Marshall, R., Taylor, S., Powers-Risius, P., and et al., B. R., "Kinetic analysis of rubidium and thallium as deposited myocardial blood flow tracers in isolated rabbit heart," Am. J. Physiol. 272, H1480-H1490 (1997).

26. Knoebel, S., Low, D., Lovelace, D., and Friedman, J., "Myocardial blood flow as measured by fractional uptake of rubidium 84 and microspheres," J. Nucl. Med. 19, 1020-1026 (1978).

27. Daniel, M., Aras, O., Smith, M., Nan, A., and Fleiter, T., "Targeted in-vivo computed tomography (CT) imaging of tissue ACE using concentrated lisinopril-capped gold nanoparticle solutions," in [Proc. SPIE], Smart Biomedical and Physiological Sensor Technologies VII 7674, 76740J (2010).

28. Cormode, D., Skajaa, T., van Schooneveld, M., and et al., R. K., "A multimodality contrast agent platform," Nano. Lett. 8, 3715-3723 (2008).

29. Langheinrich, A., Kampschulte, M., Crossmann, C., Moritz, R., W, W. R., Bohle, R., and Ritman, E., "Role of computed tomography voxel size in detection and discimination of calcium and iron deposits in atherosclerotic human coronary artery specimens," J Comput. Assist. Tomogr. 33, 517-522 (2009). 